\title{
A methodology for coupling DNS and discretised population balance for modelling turbulent precipitation
}

\author{
Hin Yan Tang ${ }^{\mathrm{a}}$, Stelios Rigopoulos ${ }^{\mathrm{a}, *}$, George Papadakis ${ }^{\mathrm{b}}$ \\ ${ }^{a}$ Department of Mechanical Engineering,Imperial College London, South Kensington \\ Campus, London $S W^{7}$ 2AZ, UK \\ ${ }^{b}$ Department of Aeronautics, Imperial College London, South Kensington Campus, \\ London SW7 2AZ, UK
}

\begin{abstract}
In this paper, we present a methodology for simulating nanoparticle formation in a turbulent flow by coupling Direct Numerical Simulation (DNS) and population balance modelling. The population balance equation $(\mathrm{PBE})$ is solved via a discretisation method employing a composite grid that provides sufficient detail over the wide range of particle sizes reached during the precipitation process. The coupled DNS/PBE approach captures accurately the strong interaction between the dynamics of turbulent mixing and particle formation processes. It also allows the calculation of the particle size distribution (PSD) of the product and enables an investigation on how it is controlled by turbulent mixing. Finally, it provides the statistics of kinetic processes and their timescales so that further analysis can be performed. The methodology is applied to the simulation of experiments of hydrodynamics and nanoparticle precipitation in a T-mixer (Schwertfirm et al., 2007, Int.
\end{abstract}

\footnotetext{
*Corresponding author

Email addresses: hin.tang16@imperial.ac.uk (Hin Yan Tang), s.rigopoulos@imperial.ac.uk (Stelios Rigopoulos), g.papadakis@imperial.ac.uk (George Papadakis)
}

Preprint submitted to International Journal of Heat and Fluid Flow November 15, 2020 
J. of Heat and Fluid Flow 28, pp. 1429-1442; Schwarzer et al., 2006, Chem. Eng. Sci. 61, pp. 167-181), and the agreement with the experimental results is very good.

Keywords: crystallization, precipitation, reacting flow, population balance, DNS, T-mixer

\section{Introduction}

A number of processes involve the formation and growth of particles within a fluid via physical or chemical mechanisms. Examples include crystallisation, atmospheric aerosols and soot formation. In these processes, the mechanisms of particle formation depend on factors such as temperature or concentration of chemical species, whose values can vary significantly within a flow field. The interaction of fluid dynamics, mixing and particle formation plays a crucial role on the process outcome and controls the properties of the particulate product. A study of these complex interactions is, however, a challenging problem, particularly in the case of turbulent flow, which induces strong temporal and spatial fluctuations in the transported species and therefore further non-linear effects on the particle dynamics.

Precipitation is a fast crystallisation process that is often driven by a chemical reaction. Reviews of precipitation can be found in Söhnel and Garside (1992), Mersmann (2001) and Myerson et al. (2019). The crystals that are formed exhibit a range of particle sizes, and the properties and quality of the product is strongly related to the particle size distribution (PSD). In most processes, precipitation occurs under turbulent flow conditions and mixing determines the local environment for chemical reaction and precipitation. 
Stirred tanks and jet impingement mixers are common types of reactors employed in such processes; the latter, in particular, have often been employed for nanoparticle precipitation due to their mixing efficiency. Achieving the right mixing conditions is thereby crucial for controlling the PSD.

Since precipitation is a fast process, it is very difficult to obtain experimentally detailed information about the local distribution of supersaturation, nucleation and growth. A numerical model coupling turbulence and precipitation dynamics could thus shed light on the underlying mechanisms at play during turbulent precipitation. The problem of modelling a particulate process involves two elements: fluid dynamics and population balance equation (PBE). The latter is a dynamic equation that describes the change in the PSD due to the particle formation processes.

Two approaches have been employed for accounting for the effects of flow and mixing on particle formation: lumped models and coupled fluid dynamics-PBE models. In the first approach, the flow effects are approximated via fully mixed or plug flow compartments, while the mixing effects are modelled via parameters. These parameters are usually averaged quantities, such as the turbulent kinetic energy and its dissipation rate, obtained either from experiments or CFD. Examples of such models can be found in Schwarzer and Peukert (2004b,a), which employs a modified version of the Engulfment-Deformation-Diffusion (EDD) model (Baldyga and Bourne, 1984a,b; Bałdyga and Bourne, 1999), and in Zauner and Jones (2000), which is based on the Segregated Feed Model (SFM) proposed by Villermaux (1989). Rigopoulos and Jones (2001, 2003a,b) proposed a methodology for coupling compartmental models with CFD and applied it on gas- 
liquid precipitation. This type of approach, however, cannot account for the strong coupling between mixing and precipitation in the case of fast reactions, in which case the space and time scales of the particle formation processes can be very small and vary considerably within each compartment.

A comprehensive account of the interplay of fluid dynamics and particle formation processes requires the direct coupling of the PBE with the equations of fluid dynamics. So far, most studies of this type are based on the Reynolds-Averaged Navier Stokes (RANS) approach (Bałdyga and Orciuch, 2001; Gavi et al., 2007a; Di Veroli and Rigopoulos, 2010; Wu et al., 2017). When averaging is applied to the PBE, however, several unclosed terms appear in the nucleation and growth terms; these terms were systematically studied by Rigopoulos (2007), where a PDF approach was also proposed for modelling them. Subsequently, Di Veroli and Rigopoulos (2009, 2010) applied the RANS-PBE-PDF approach to turbulent precipitation. In the latter of these works, it was demonstrated that the unknown fluctuations may have competing effects that can lead to erroneous conclusions when neglected. Other works have employed RANS closures based on presumed PDF methods (Bałdyga and Orciuch, 1997, 2001), where the shape of the PDF is presumed rather than calculated.

In recent years, the application of Large Eddy Simulation (LES) to chemical reactions in the liquid phase and to precipitation has started to be explored. In the case of very fast and localised reactions, such as combustion and precipitation, subgrid models for turbulence-chemistry and turbulenceparticle formation interaction are required, as the reactions can occur at the subgrid scales. Makowski and Bałdyga (2011) conducted an LES of pre- 
cipitation with a presumed PDF subgrid closure akin to the model used in Bałdyga and Orciuch (1997) in the context of RANS. The extension of the transported PDF method to LES was first proposed by Gao and O'Brien (1992) and was subsequently explored in combustion. In liquid flows, van Vliet et al. (2005) investigated a parallel-competitive reaction system, while van Vliet et al. (2007) investigated a polymerisation reaction; in both studies, a subgrid probability density function (PDF) was employed in conjunction with the Interaction with the Mean (IEM) mixing model. For turbulent reacting flows with particle formation, interactions between turbulence and particle formation processes such as nucleation and growth at the subgrid scales are possible. To account for these interactions, the PBE-PDF method was extended to LES by Sewerin and Rigopoulos (2017b), where it was applied to aerosol condensation. Subsequent works applied the LES-PBE-PDF to soot formation (Sewerin and Rigopoulos, 2018, 2019).

The modelling of turbulent precipitation via DNS and PBE was first carried out by Schwarzer et al. (2006), Gradl et al. (2006) and Gradl and Peukert (2009). In these studies, it was shown that the coupling of DNS and PBE allows considerable insights into the interaction of fluid dynamics and particle formation processes. However, the PBE was solved in the form of a post-processing step along trajectories of fluid elements. The information provided from the DNS to the PBE post-processor included the local energy dissipation and the evolution of a passive scalar, and was used for the computation of the parameters of a mixing model (a modified version of the EDD model) that was applied along the trajectories. Apart from the question of statistical convergence (700 trajectories were computed), an issue with this 
approach is the lack of direct calculation of the coupling between the particle and liquid phase via species consumption.

The solution of the PBE also poses considerable challenges. Various methods have been proposed, a recent review of which can be found in Rigopoulos (2019), but the methods that have been coupled with CFD in turbulent flows are mainly the moment methods and the discretisation methods. The former, initially introduced by Hulburt and Katz (1964), involves solving equations for the evolution of the moments of the distribution. The moments are integral properties of the PSD and some of them have a physical meaning for example, when a distribution with respect to volume is considered, the zeroth and first moment represent the total number and total volume of particles, respectively. The use of moment methods is motivated by the reduced memory and CPU requirements that they incur, as the number of variables is reduced. However, the PSD is not predicted and assumptions must be made for reconstructing it from the moments. Furthermore, the moment equations are unclosed unless the functions representing the particle dynamics (such as growth and aggregation) assume specific forms. Several approaches have been proposed to obtain closure for the moment equations, such as series expansions, presumed distributions, moment interpolation and quadrature methods. Among these, methods in the last category have found considerable application to precipitation. These methods originate in the Quadrature Method of Moments (QMOM) (McGraw, 1997), where the distribution is regarded as the weight function and the remaining part of the integrand as the function to be integrated. In a further development, in the Direct Quadrature method of moments (DQMOM) (Marchisio and Fox, 2005), ODEs are 
derived for obtaining the quadrature parameters directly. This approach was employed in the modelling of precipitation by Gavi et al. (2007b) and Metzger and Kind (2017).

Discretisation methods, on the other hand, discretise the PBE into intervals, or sections (hence they are also called 'sectional methods'). They do not have the closure issues of moment methods and predict directly the distribution, rather than its moments. While the CPU requirements are higher, the PBE equations are not stiff and their solution can take only a small fraction of the CPU time Liu and Rigopoulos (2019). Methods have also been developed for addressing the two main challenges in discretisation methods. The first one is the numerical diffusion in the growth term, which can be mitigated with a high-resolution scheme (Qamar et al., 2006) or virtually eliminated with the recently developed explicit adaptive grid method (Sewerin and Rigopoulos, 2017a). A hybrid Monte Carlo - discretisation method Bouaniche et al. (2019) has also been proposed that overcomes this issue. The second challenge is the conservation of moments in the integral aggregation terms, for which several approaches have been developed; a review of these can be found in Rigopoulos (2019).

In the present work, we present a methodology for coupling DNS of the flow field with a discretised population balance approach for solving the PBE. This approach has the following advantages: a) the flow field is fully resolved, thus accounting for all the temporal and spatial scales of turbulence, b) the population balance is solved without closure assumptions, c) the detailed PSD is predicted, and d) the DNS and PBE are directly coupled via the source terms in the transport equations. The numerical aspects of the 
method, namely flow field simulation, convergence of PBE numerical solution and source term coupling, are validated on their own before the presentation of the results of the coupled approach. By preventing the compensation of numerical and modelling errors, this approach will allow the investigation of the effects of turbulent mixing on the PSD and the evaluation of the sources of uncertainties. In addition, it should serve as a future model development and validation platform.

\section{The DNS-PBE methodology}

\subsection{Fluid dynamics and DNS}

We assume incompressible flow and solve the continuity and NavierStokes equations, which are shown below in Cartesian tensor notation:

$$
\begin{gathered}
\frac{\partial u_{i}}{\partial x_{i}}=0 \\
\frac{\partial u_{i}}{\partial t}+\frac{\partial\left(u_{j} u_{i}\right)}{\partial x_{j}}=-\frac{1}{\rho} \frac{\partial p}{\partial x_{i}}+\frac{\mu}{\rho} \frac{\partial^{2} u_{i}}{\partial x_{j} \partial x_{j}}
\end{gathered}
$$

where $u_{i}$ is the velocity in $i$-th direction, $p$ is the static pressure, $\rho$ is the fluid density and $\mu$ is the dynamic viscosity. The last two quantities are assumed to be constant, as the mass fraction of ions in water is small.

The DNS is performed with our in-house code PANTARHEI, which has been used extensively to simulate transitional and turbulent flows in boundary layers, around airfoils, behind fractal grids and inside stirred vessels (Xiao and Papadakis, 2017, 2019; Thomareis and Papadakis, 2017, 2018; Paul et al., 2018; Başbuğ et al., 2018). The Navier-Stokes equations are discretised in physical space using the finite volume method in a collocated, unstructured grid. The fractional step method is used to extract pressure and correct 
velocities to satisfy the continuity equation. The Rhie and Chow (1983) interpolation scheme is employed to compute the velocities at the faces of the control volumes. Second order central approximation is used for both convection and diffusion terms. For the transient terms, a third-order backward difference scheme (BDF3) is employed:

$$
\left(\frac{\partial \phi}{\partial t}\right)^{n+1}=\frac{1}{6 \Delta t}\left(11 \phi^{n+1}-18 \phi^{n}+9 \phi^{n-1}-2 \phi^{n-2}\right)
$$

The orthogonal diffusion terms are treated implicitly, while the convection and non-orthogonal diffusion terms are treated explicitly, using third-order extrapolation (EXT3):

$$
\phi^{n+1}=3 \phi^{n}-3 \phi^{n-1}+\phi^{n-2}
$$

The numerical scheme is inviscidly stable up to a CFL number of 0.634 , Fishpool and Leschziner (2009). The matrix coefficients remain constant and are reused in every time step. The resultant discretised equations are solved using the Krylov subspace method GMRES implemented within the PETSc library (Abhyankar et al., 2018; Balay et al., 2019). BoomerAMG from the Hypre package (Falgout and Yang, 2002) is employed as an algebraic multigrid preconditioner for the matrices of velocity and pressure correction.

\subsection{PBE formulation}

The PBE is formulated in terms of the number density of particles per unit volume of particle and unit volume of solution, i.e. $n(v ; \vec{x}, t) d v$ is the concentration of particles with volume between $v$ and $v+d v$ at point $\vec{x}$ and time $t$. For brevity, the dependence of variables on $v, \vec{x}$ and $t$ will be omitted. The PBE is: 


$$
\frac{\partial n}{\partial t}+\frac{\partial\left(u_{i} n\right)}{\partial x_{i}}+\frac{\partial(G(C, v) n)}{\partial v}=\frac{\mu}{S c} \frac{\partial^{2} n}{\partial x_{i} \partial x_{i}}+B(C) \delta\left(v-v_{0}\right)
$$

where $B(C)$ and $G(C, v)$ are the nucleation and growth rates respectively. Both are functions of the composition, and the growth rate is also a function of particle volume, as we will see in Sec. 3. Nucleation takes place at the size of the nuclei, $v_{0}$, and is thus represented by a delta function. The PBE may include further terms for processes such as aggregation and breakage, but these are not present in the experiment simulated (Schwarzer et al., 2006) here. It must be noted that, when aggregation is not present, it is customary to express the PBE in terms of a linear size dimension, such as particle diameter. Our PBE solver has been developed to deal also with aggregation (Liu and Rigopoulos, 2019), which is better captured in the volume domain because aggregation events conserve particle volume. While there is no aggregation in the experiments simulated here, the formulation in terms of particle volume is retained; this does not make any difference, as the two formulations are equivalent. The volume- and diameter-based number density are related as follows:

$$
n=n_{L} \frac{d L}{d v}=n_{L} \frac{1}{3} k_{v}^{-\frac{1}{3}} v^{-\frac{2}{3}}
$$

The dependence of nucleation and growth rates on the concentrations of chemical species means that the PBE must be coupled with the species transport equation:

$$
\frac{\partial C_{\alpha}}{\partial t}+\frac{\partial\left(u_{i} C_{\alpha}\right)}{\partial x_{i}}=\frac{\mu}{\operatorname{Sc}} \frac{\partial^{2} C_{\alpha}}{\partial x_{i} \partial x_{i}}+R_{\alpha}
$$




\subsection{PBE discretisation}

The numerical solution of the PBE is carried out with our in-house solver CPMOD. The PBE is discretised in the particle volume space to yield a set of coupled partial differential equations for the discretised number densities, $n_{k}:$

$$
\frac{\partial n_{k}}{\partial t}+\frac{\partial\left(u_{i} n_{k}\right)}{\partial x_{i}}+\frac{\partial\left(G(C, v) n_{k}\right)}{\partial v}=\frac{\mu}{S c} \frac{\partial^{2} n_{k}}{\partial x_{i} \partial x_{i}}+B(C) \delta\left(v-v_{0}\right)
$$

The transient and transport terms in the discretised PBE are treated numerically with the same BDF3-EXT3 scheme employed for the momentum equations, except that all terms are treated explicitly. The Gamma differencing scheme by Jasak et al. (1999) is employed to preserve boundedness of the solution. This scheme blends the upwind and central differencing schemes based on the ratio of two consecutive differences, $\tilde{\phi}$ (Eq. 9). The evaluation of this ratio usually requires upstream quantities, which imposes challenges in the context of unstructured grids. In the method of (Jasak et al., 1999) the ratio can be easily computed with knowledge of the quantities in the local $(\phi)$ and downstream cell $\left(\phi_{D}\right)$, the gradient at the local cell $\left(\nabla \phi=\frac{d \phi}{d x_{i}}\right)$, as well as the vector between two cell centroids $(\vec{d})$ :

$$
\tilde{\phi}=\frac{\phi-\phi_{U}}{\phi_{D}-\phi_{U}}=1-\frac{\phi_{D}-\phi}{2\left(\frac{d \phi}{d x_{i}}\right) d_{i}}
$$

The blending factor $\gamma$ serves as a controlling parameter on the scheme weighting:

$$
\gamma=\frac{\tilde{\phi}}{\beta_{m}}
$$

We use the value 0.1 for the blending coefficient $\beta_{m}$ in order to maintain a sharp profile (Jasak et al., 1999). This makes the scheme suitable for unstructured grids. 
The nucleation and growth terms in the transported PBE are treated as additional source terms. They are discretised with a finite volume method in the particle volume domain. The nucleation rate, $B_{N}$, is a source of particle number (rather than number density) taking place at the interval of the nuclei:

$$
B_{N}(C)=B(C) d v_{0}
$$

The growth term, however, is a first-order derivative, akin to a convection term in the particle volume domain, and represents a propagation mechanism. Therefore, care must be taken in its numerical treatment to avoid spurious oscillations. In this work, the growth term is discretised as in Eq. 12 that includes the growth flux $G(C) n$ from the adjacent intervals.

$$
\left(\frac{\partial G(C, v) n}{\partial V}\right)_{k}=\frac{1}{\Delta V_{k}}\left[(G(C) n)_{k^{+}}-(G(C) n)_{k^{-}}\right]
$$

A Total Variation Diminishing (TVD) scheme (Qamar et al., 2006) is implemented to prevent oscillations in the PSD. The scheme employs piecewise polynomial interpolation to obtain a weighted blend of upwind and central scheme (Eq. 13).

$$
(G(C) n)_{k^{+}}=G_{k^{+}}\left[n_{k}+\frac{1+\kappa}{4}\left(n_{k+1}-n_{k}\right)+\frac{1-\kappa}{4}\left(n_{k}-n_{k-1}\right)\right]
$$

The blending is governed by a parameter $\kappa \in[-1,1]$ and the monotonicity is preserved by introducing a limiter function. In this work, we employ the limited $\kappa=1 / 3$ scheme (Eq. 14) with the flux limiting function $\phi\left(r_{k}^{+}\right)$by Koren (1993) (Eq. 15 and Eq. 16), yielding second order accuracy. The growth flux can therefore be evaluated by:

$$
(G(C) n)_{k^{+}}=G_{k^{+}}\left[n_{k}+\frac{1}{2} \phi\left(r_{k}^{+}\right)\left(n_{k}-n_{k-1}\right)\right],
$$


where the flux limiting function and its limiter $r_{k}$ are:

$$
\begin{gathered}
\phi\left(r_{k}^{+}\right)=\max \left[0, \min \left[2 r_{k}^{+}, \min \left(\frac{1}{3} \frac{\Delta v_{k}}{\Delta v_{k^{-}}}+\frac{2 r_{k}^{+}}{3} \frac{\Delta v_{k}}{\Delta v_{k^{+}}}, 2\right)\right]\right] \\
r_{k}^{+}=\frac{n_{k+1}-n_{k}+\varepsilon}{n_{k}-n_{k-1}+\varepsilon}
\end{gathered}
$$

The growth rate in Eq. 14 is evaluated at the right nodal point of the $k^{\text {th }}$ interval whereas the number densities are taken from the mid-point of the corresponding interval. The chosen scheme can account for size-dependent growth kinetics, and the flux limiter is suitable for a non-equidistant PBE grid, such as the one employed here. For more details on the scheme, one may refer to Qamar et al. (2009) and Gunawan et al. (2004).

In addition to the mass conservation in the particle size domain, the mass consumption of ions during reaction must be consistent with the generation of particle mass. To ensure this, we compute the reaction source term in Eq. 7 from the rate of increase of particle volume in the PBE. The total particle volume is given by the first moment of the PSD:

$$
M_{1}=\int_{0}^{\infty} v n(v) d v
$$

The total rate of increase of the first moment due to particle formation can be obtained by summing the volume of nuclei formed and the increase of volume in every interval in the discretised PBE (Eq. 8) due to growth, as shown in below:

$$
\left.\frac{d M_{1}}{d t}\right|_{\text {source }}=\left[B(C) v_{0}+\sum_{k=1}^{m} v_{m, k}\left(\frac{\partial G\left(C, v_{k}\right) n_{k}}{\partial v}\right) d v_{k}\right]
$$

where the subscript 'source' is used to distinguish this rate from the rate of change due to transport. The first moment can be converted to concentration 
of the chemical species that the crystal is composed of as follows:

$$
C_{c}=\frac{\rho_{c} M_{1}}{M W_{c}}
$$

The reaction rate in the species transport equations (Eqs. 7) can thus be calculated from the rate of change of the crystal species and the corresponding stoichiometric factor:

$$
R_{\alpha}=\left.\frac{\nu_{\alpha} \rho_{c}}{M W_{c}} \frac{d M_{1}}{d t}\right|_{\text {source }}
$$

Both the reaction and the PBE source term are discretised with the EXT3 extrapolation scheme in order to match the time-marching strategy of the transport equations. The consistence of source terms in the PBE and scalar transport equations will be validated in Sec. 4.3.2.

The grid employed for the discretisation of the PBE in the particle volume domain is crucial for the accuracy of the numerical approach in a nucleationgrowth problem. The grid has to cover a large domain, while avoiding numerical diffusion. In addition, the small nuclei size and size-dependent growth rate require a fine resolution at the low end of the particle volume spectrum. Finally, the number of discretised number densities must be kept to a minimum in order to keep the CPU requirements manageable. Uniform and geometric grids are the simplest types of grid employed for the PBE. A uniform fine grid offers very good resolution but is too computationally expensive, while a geometric grid is well suited to fast-growing particles but can result in numerical diffusion at large intervals. More advanced methods, such as an adaptive grid approach (Sewerin and Rigopoulos, 2017b, 2018), have been employed to maintain good grid resolution with very few grid intervals in LES simulations. 
Considering the computational demands of DNS, in this study we propose a fixed grid approach that features minimal numerical diffusion. This approach combines the advantages of the uniform and geometric grid into a composite grid, as illustrated in Fig. 1. The grid is made for particles ranging from $0.6 \mathrm{~nm}$ and $500 \mathrm{~nm}$ with a total of 45 intervals. The smallest scale is chosen to accommodate the very small nuclei that form in regions of high supersaturation. The particle range is divided into 3 sections. A high-resolution geometric grid is used to discretise a narrow range of scales over the fast-growing zone $(0.6 \mathrm{~nm}$ to $20 \mathrm{~nm})$, intended to capture the fastgrowing nuclei. Subsequently, a uniform grid is used for an intermediate size range (20 $\mathrm{nm}$ to $150 \mathrm{~nm}$ ) to provide reasonable resolution for this range that accounts for the major part of the final distribution. Finally, the last section is discretised with a coarse geometric grid over a very wide range. The role of this section is to accommodate the largest particles and avoid a spurious mass loss.
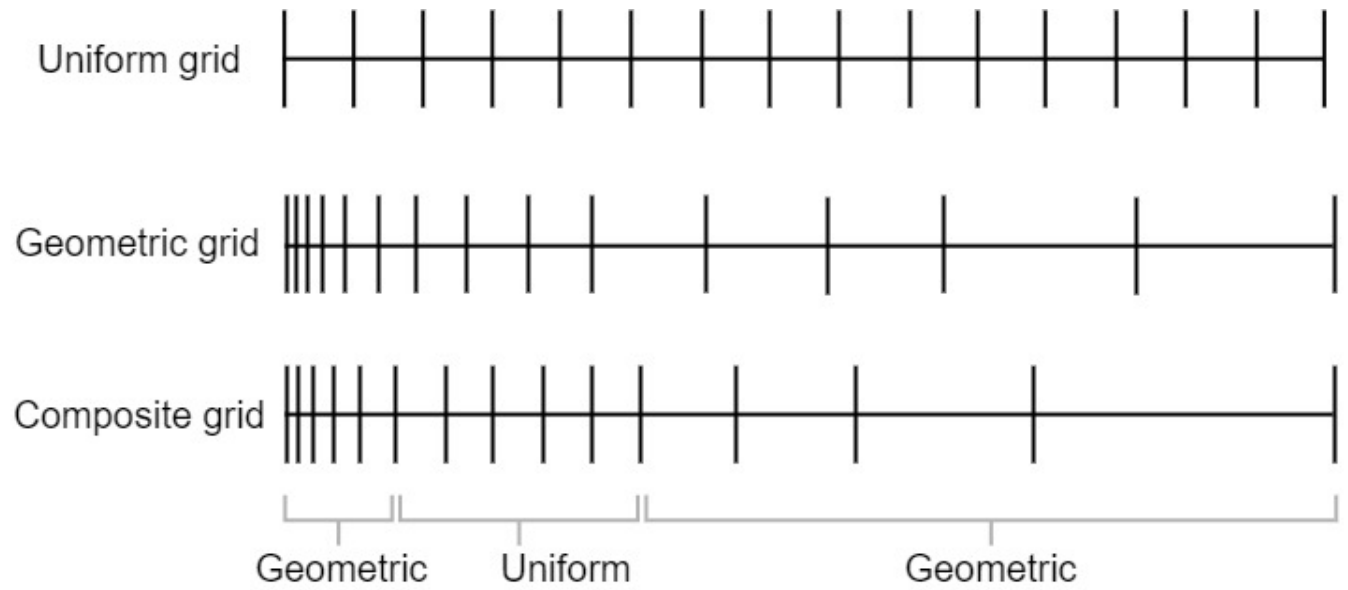

Figure 1: Illustration of the composite grid, compared with the uniform and geometric grids. 


\subsection{Coupling of DNS and PBE}

In the proposed method, each cell in the DNS simulation has its own local compositions and PSD. The transport of species and particles in each cell depends on the local velocity and is fully resolved by DNS. The inertia of nanoparticles is negligible and they can be assumed to follow the streamlines of the flow. The PBE is coupled to the species transport equations via the reactant consumption. The coupling scheme is illustrated in Fig. 2.

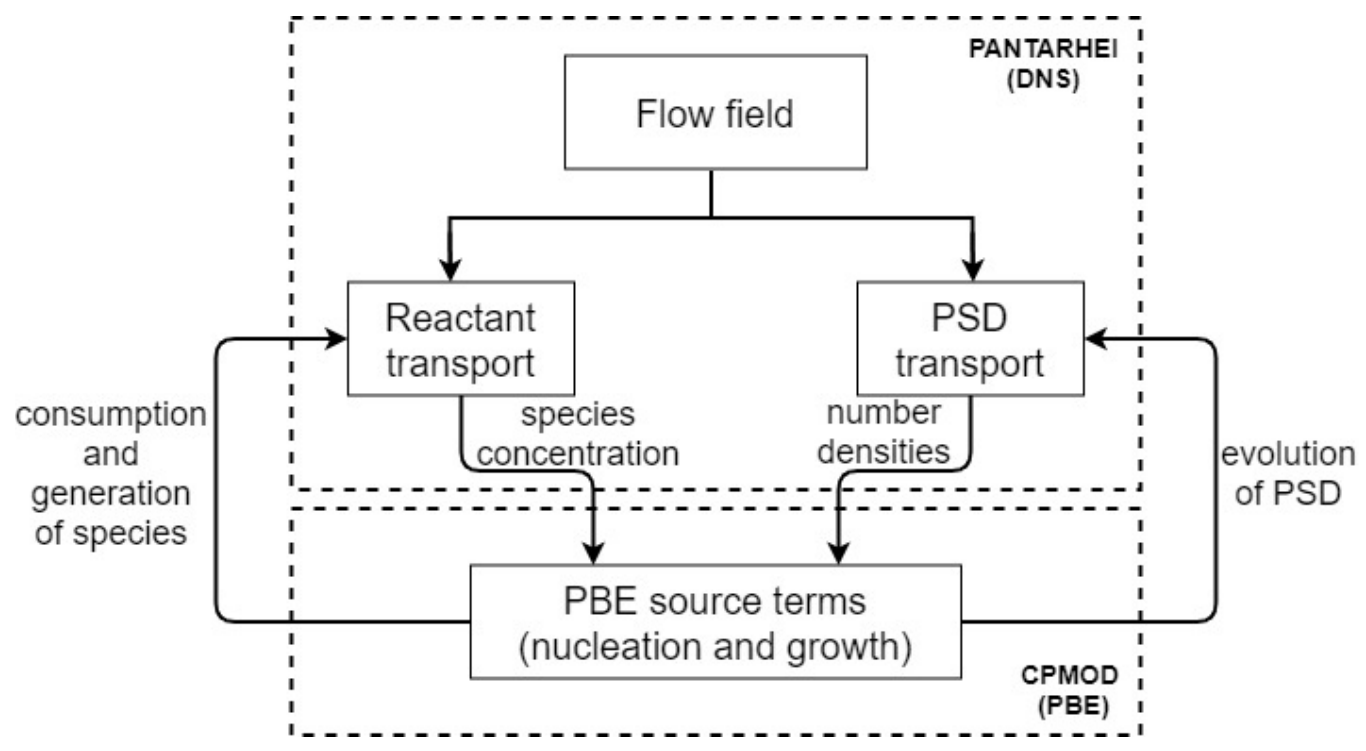

Figure 2: Graphical representation of the proposed coupling of PBE, flow field and species transport.

Two issues pose a challenge to simulations of turbulent precipitation, as they cannot be resolved via DNS using current computing hardware. The first issue is the mixing at the sub-Kolmogorov, or Batchelor, scales, while the second one is the Schmidt number, $S c$, which can be as high as 1000 . These two issues are related but distinct, and are more likely to be of importance in 
the species transport equation, due to the sensitivity of nucleation and growth rates on mixing, rather than in the PBE. This is a major unresolved problem in reacting flows in the liquid phase that has been discussed extensively by Bałdyga and Bourne (1999). The few studies so far investigating effects of high $S c$ have been primarily on inert scalars. For example, these effects on scalar transport were investigated numerically by Derksen (2012) through grid refinement. To our knowledge, however, such studies have not been carried out in reacting flows and precipitation.

The simulation with the grid of 21 million cells employed in this study has maximum grid-to-Kolmogorov scale ratio $\left(\Delta x / \eta_{K}\right)$ of about 2 (see Sec 4.2). The ratio of Kolmogorov $\left(\eta_{K}\right)$ to Batchelor $\left(\eta_{B}\right)$ scales varies as $\sqrt{S c}$, so for $S c=1000, \eta_{B}$ would then be $\sqrt{1000} \approx 31$ times smaller than $\eta_{K}$. In order to maintain a ratio $\left(\Delta x / \eta_{B}\right)$ of about 2 , the grid spacing $\Delta x$ should be around 30 times finer in each direction, which corresponds to $30^{3}=27,000$ times more cells than the current grid, i.e. 570 billion cells, which is not possible with the current or even foreseeable computational resources. Therefore, a modelling choice has to be made in this respect. The use of high $S c$ in the simulation poses a computational challenge on its own right, because a second order central scheme would require a TVD approach to suppress numerical oscillations, which would amount to artificial diffusion. On the other hand, the employment of a high-order scheme in a grid that does not resolve the Batchelor scales is also problematic and would result in neglecting the extra diffusion induced by the latter. In the current simulation we have employed $S c=1$, which compensates to some extent for the diffusion in the Batchelor scales. An alternative choice would be to employ a micromixing model, in a 
manner similar to LES studies (van Vliet et al., 2005; van Vliet et al., 2007; Makowski and Bałdyga, 2011). However, this choice in the context of a DNS study is questionable, because in LES these models account for mixing in the inertial and Kolmogorov scales as well, which are already accounted for in the DNS simulation. In the DNS approach of Schwarzer et al. (2006), Gradl et al. (2006) and Gradl and Peukert (2009), a micromixing model was employed because the PBE was applied as a post-processing step along Lagrangian trajectories, unlike the present study where the coupling between the PBE, fluid flow and species transport is direct. The use of $S c=1$ is therefore a reasonable modelling choice, subject to validation of the simulation outcome with experimental results.

The time step used in the coupled simulation is different from the standalone DNS, as a smaller step size is required due to the fast precipitation timescales. Therefore, apart from the CFL condition, an additional condition should be met for a stable solution. As has been discussed previously, the growth term appearing in the PBE acts like a convection in the particle size domain, hence a criterion similar to CFL can be written as in Eq. 21 (Gunawan et al., 2004). We refer to this criterion as $\mathrm{CFL}_{P B E}$ in the following in contrast to the $\mathrm{CFL}_{F L O W}$ for the flow solution. This additional criterion imposes a stricter condition on the time step,

$$
\max \left|\frac{\left(G_{k}\right) \Delta t}{\Delta L_{k}}\right| \leq 1
$$

where $\Delta L_{k}$ is the distance between two neighbouring grid points in the PBE grid. To show the necessity and importance of meeting the $\mathrm{CFL}_{P B E}$ criterion, several coupled simulations were conducted with different time step sizes. All 
cases meet the $\mathrm{CFL}_{F L O W}$ criterion but had different $\mathrm{CFL}_{P B E}$. An integral quantity from each case is compared to evaluate convergence. Here we use the total number of particles in the domain, which is obtained by summing the zeroth moment in all cells, and monitor how it evolves with time. The comparison is shown in Fig. 3, where solid lines and dashed lines are used for cases with $\mathrm{CFL}_{P B E}$ smaller than and larger than unity respectively. The plot clearly shows that as long as $\mathrm{CFL}_{P B E}$ is less than 1, the evolution of the number of particles gradually increases with similar trend. In contrast, the number of particles in large $\mathrm{CFL}_{P B E}$ cases suddenly diverge after the start of the precipitation process and the diverging point begins earlier for larger $\mathrm{CFL}_{P B E}$. This is caused by the overconsumption in the ions due to large time step. Thus, it is crucial that the time increment meets the CFL condition in both convection and growth. In the precipitation simulations, the time step is chosen in such a way as to yield a $\mathrm{CFL}_{P B E}$ of 0.35 , which corresponds to $1.5 \cdot 10^{-7} \mathrm{~s}$.

\section{Precipitation Kinetics}

The system that will be studied in this work is the precipitation of $\mathrm{BaSO}_{4}$, according to the following reaction:

$$
\mathrm{BaCl}_{2}+\mathrm{H}_{2} \mathrm{SO}_{4} \rightarrow \mathrm{BaSO}_{4}+2 \mathrm{HCl}
$$

The kinetics have been studied extensively and several kinetic models can be found in the literature. However, most of them are applicable over a limited range of conditions. Therefore, the kinetics in this study are based on classical theories of nucleation and growth, along the lines of Schwarzer et al. (2006). 


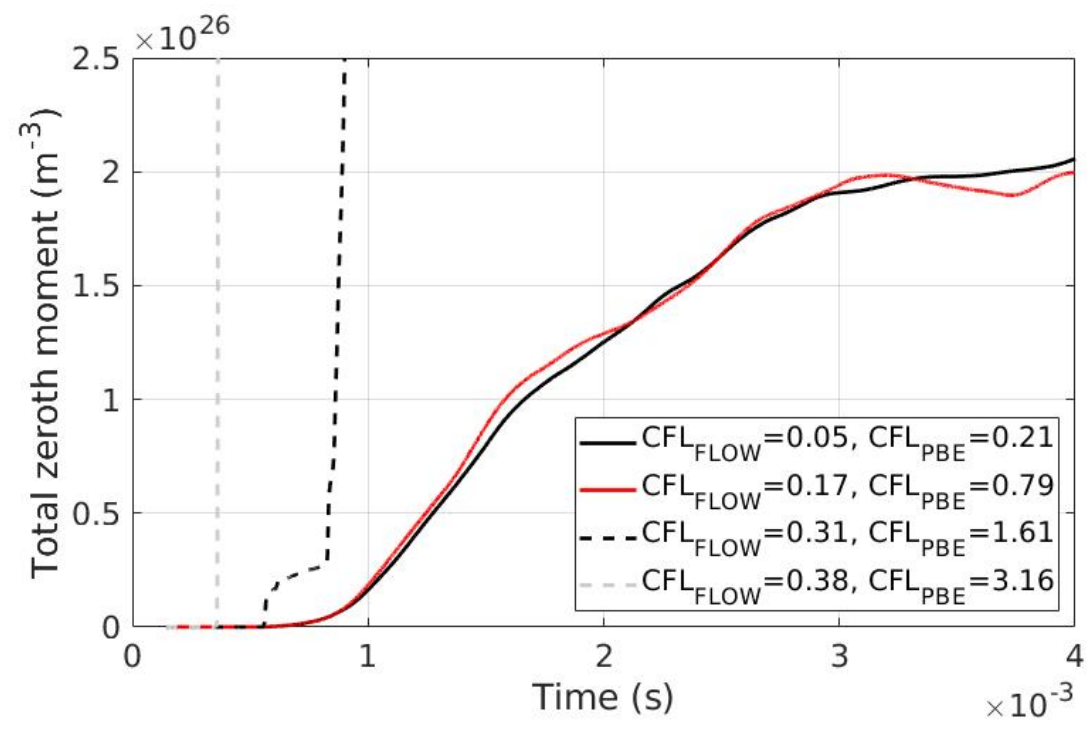

Figure 3: Convergence study on the effect of time step under different $\mathrm{CFL}_{P B E}$ values. The evolution of the total zeroth moment in the whole domain is plotted against time.

The kinetic expressions are suggested by Mersmann (2001) and have also been used by several more authors including Gavi et al. (2007b), Marchisio et al. (2006) and Metzger and Kind (2017) under high supersaturation conditions, as these encountered here.

Supersaturation is the driving force for nucleation and growth, and it is expressed as follows:

$$
S=\gamma_{ \pm} \sqrt{\frac{C_{B a_{\text {free }}^{2+}} C_{S O_{\text {free }}^{2-}}}{K_{S P}}}
$$

where $K_{S P}$ is the solubility product. The free ion concentration is used when calculating the supersaturation, due to the incomplete dissociation of $\mathrm{H}_{2} \mathrm{SO}_{4}$ and ion complex formation of $\mathrm{BaSO}_{4}$. The equilibrium constants for $\mathrm{HSO}_{4}{ }^{-}$ ion dissociation and for $\mathrm{BaSO}_{4}$ complex formation are chosen according to Schwarzer and Peukert (2004a). 
The growth kinetics are calculated according to Gradl and Peukert (2009). For the conditions in this study, the growth is transport-controlled, and the expression employed is:

$$
G_{L}=\frac{d L}{d t}=\frac{k_{a}}{3 k_{v}} \frac{S h D_{A B} \sqrt{K_{S P}} M W_{c}(S-1)}{\rho_{C} L}
$$

where $S h$ is the Sherwood number, taken to be 2 as in Schwarzer and Peukert (2004a). Note that this expression yields a linear growth rate, which can be converted into a volumetric growth rate for use in the volume-based PBE (Eq. 25). The conversion is based on the change of variable from $L$ to $v$, $d v=3 k_{v} L^{2} d L$, and requires a shape factor, $k_{v}$. From the morphology shown in the experiments (Schwarzer and Peukert, 2002), the particles have an ovallike shape that is close to spherical. Therefore we employ the surface and volume shape factors for spherical particles $k_{a}=\pi$ and $k_{v}=\frac{\pi}{6}$, with the particle surface area and volume defined as $k_{a} L^{2}$ and $k_{v} L^{3}$ respectively.

$$
G=\frac{d v}{d t}=3 k_{v}^{\frac{1}{3}} G_{L} v^{\frac{2}{3}}
$$

In terms of particle volume, Eq. 25 can be written as:

$$
G=\frac{d v}{d t}=\frac{k_{a}}{k_{v}^{\frac{2}{3}}} \frac{S h D_{A B} \sqrt{K_{S P}} M W_{c}(S-1)}{\rho_{C}} v^{\frac{2}{3}}
$$

According to Schubert (1998), primary homogeneous nucleation is the dominant nucleation mechanism at high supersaturation. The homogeneous nucleation rate is given by expression Eq. 27, according to the classical nucleation theory (Mersmann, 2001):

$$
B_{N}=1.5 D_{A B}\left(\sqrt{K_{S P}} S N_{A}\right)^{\frac{7}{3}} \sqrt{\frac{\gamma_{C L}}{k T}} V_{m} \exp \left(-\frac{16}{3}\left(\frac{\gamma_{C L}}{k T}\right)^{3} \frac{V_{m}^{2}}{(\nu \ln S)^{2}}\right)
$$




\section{Results and discussion}

The methodology will now be applied to the simulation of $\mathrm{BaSO}_{4}$ precipitation in a T-mixer. The flow field simulation, convergence of PBE numerical solution and source term coupling will all be validated individually before the presentation of the results of the coupled approach for the prediction of the particle size distribution.

\subsection{Geometry and DNS parameters}

Two experiments are simulated in this paper. The first one investigates only the hydrodynamics of the T-mixer and will be used for flow validation. This experiment was conducted at Reynolds number 500 (without precipitation) was documented in Schwertfirm et al. (2007). The T-mixer in this case contains a square channel of $80 \mathrm{~mm}$ width with two $\phi 40 \mathrm{~mm}$ inlets. This is an exact scale-up (80 times) of the one used in the precipitation experiments; this scale was chosen to enable PIV measurements.

The second experiment involves the precipitation of $\mathrm{BaSO}_{4}$ nanoparticles in a T-mixer and was conducted by Schwarzer and Peukert (2002), Schwarzer and Peukert (2004b), Schwarzer and Peukert (2004a), Schwarzer et al. (2006) and Gradl et al. (2006). This experiment is simulated with the coupled DNS-PBE method. The case simulated is mixer \#5 in Schwarzer et al. (2006), with Reynolds number 1135. This T-mixer consists of two $\phi 0.5 \mathrm{~mm}$

inlet pipes with a $10 \mathrm{~mm}$ long and $1 \mathrm{~mm}$ width square cross-section mixing channel. The setup of the precipitation experiment is illustrated in Fig. 4 and the operating conditions of this mixer are listed in Tab. 1, yielding a nominal supersaturation (i.e. a supersaturation that would result from a 
perfect mixing of the two streams) of approximately 1000. In the conditions of this experiment, no particle aggregation was present. This is due to the $\mathrm{Ba}^{2+}$ excess, which prevents aggregation due to repulsive electrostatic forces (Eble, 2000; Kucher et al., 2006; Schwarzer and Peukert, 2005).

In both the flow-only and the precipitation case, the flow rate at the one inlet is equal to the one at the other inlet. The Reynolds number is defined at the mixing channel as follows:

$$
R e=\frac{\rho u_{M} H}{\mu}
$$

where $u_{M}$ and $H$ are the mean velocity and width in the mixing channel respectively as indicated in Fig. 4. The flow can be considered turbulent at Reynolds number larger than 400 (Telib et al., 2004). The mixing channel is aligned with the z-axis, while the feed pipes are along the x-axis, as shown in Fig. 4. The grid is Cartesian and contains 19 and 21 million cells in the flow-only and precipitation cases respectively, the vast majority of which (18.6 and 19.5 million cells respectively) are in the mixing channel. The grid spacing is uniform $(\Delta x=0.00875 H$ and $\Delta x=0.008 H$ respectively) throughout the channel. This spacing is comparable to the finest one in the simulations of Schwertfirm et al. (2007) and Schwarzer et al. (2006) $(\Delta x=$ $0.011 H, \Delta y=0.005 H, \Delta z=0.005 H)$. Poiseuille flow is assumed at both inlets, and a convective boundary condition is used at the exit plane:

$$
\frac{\partial u_{i}}{\partial t}+u_{3} \frac{\partial u_{i}}{\partial z}=0
$$

The cell sizes are selected to be of the order of Kolmogorov length scale, as will be demonstrated in Sec. 4.2 
Table 1: Inlet Ion Concentrations

\begin{tabular}{lll}
\hline Ion species & $\begin{array}{l}\mathrm{C}_{\text {inlet }} \\
\left(k m o l / m^{3}\right)\end{array}$ & Inlet \\
\hline $\mathrm{Ba}^{2+}$ & 0.5 & 1 \\
$\mathrm{Cl}_{2}{ }^{-}$ & 1 & 1 \\
$\mathrm{H}^{+}$ & 0.33 & 2 \\
$\mathrm{HSO}_{4}{ }^{-}$ & 0.33 & 2 \\
\hline
\end{tabular}

In the flow-only simulation, the flow statistics were collected after reaching a statistically steady state. In the precipitation simulation, a flow-only DNS simulation was performed for ten flow-through times before injecting the reactants. This was to ensure that the flow field had reached a statistically steady state before the onset of the precipitation process. The time steps used for the flow field in the flow-only simulation and the initial flow-only part of the precipitation simulation were $3 \cdot 10^{-3} \mathrm{~s}$ and $3 \cdot 10^{-7} \mathrm{~s}$ respectively, which correspond to $\mathrm{CFL}_{F L O W}=0.3$. In the precipitation simulation, the time step was afterwards reduced to $1.5 \cdot 10^{-7} \mathrm{~s}$ as mentioned in Sec. 2.4, in order to satisfy the $C F L_{P B E}$ condition.

\subsection{Flow field}

In this section, we present the results from the simulation of the flow field for the larger T-mixer experiment (Schwertfirm et al., 2007); this simulation was performed in order to validate the hydrodynamics. Fig. 5 shows the grid-size-to-Kolmogorov-scale ratio in the whole domain. It can be seen that this ratio is less than 1.5 in most of the domain and the maximum ratio is 2.3, 

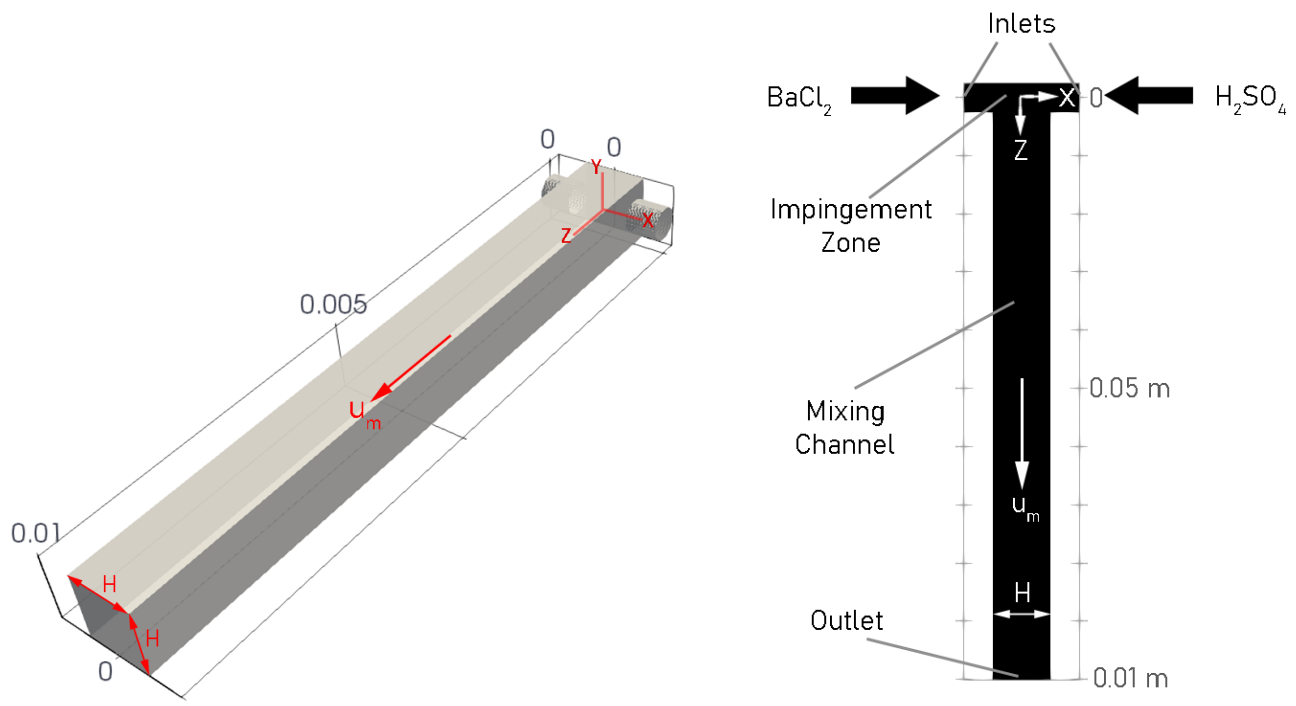

Figure 4: Illustration of the T-mixer.

so it can be concluded that the flow field is well resolved (Moin and Mahesh, 1998). The maximum values are located in the region of highest dissipation, which is on the bottom wall of the impingement region.

Fig. 6 shows the mean flow streamlines. The two impinging streams merge and produce a large helical vortex in the mixing channel, that provides a global mixing environment. The helical vortex is clearly presented in the mean velocity vector plot in Fig. 7. This global feature is in visual agreement with that reported in Schwertfirm et al. (2007). 


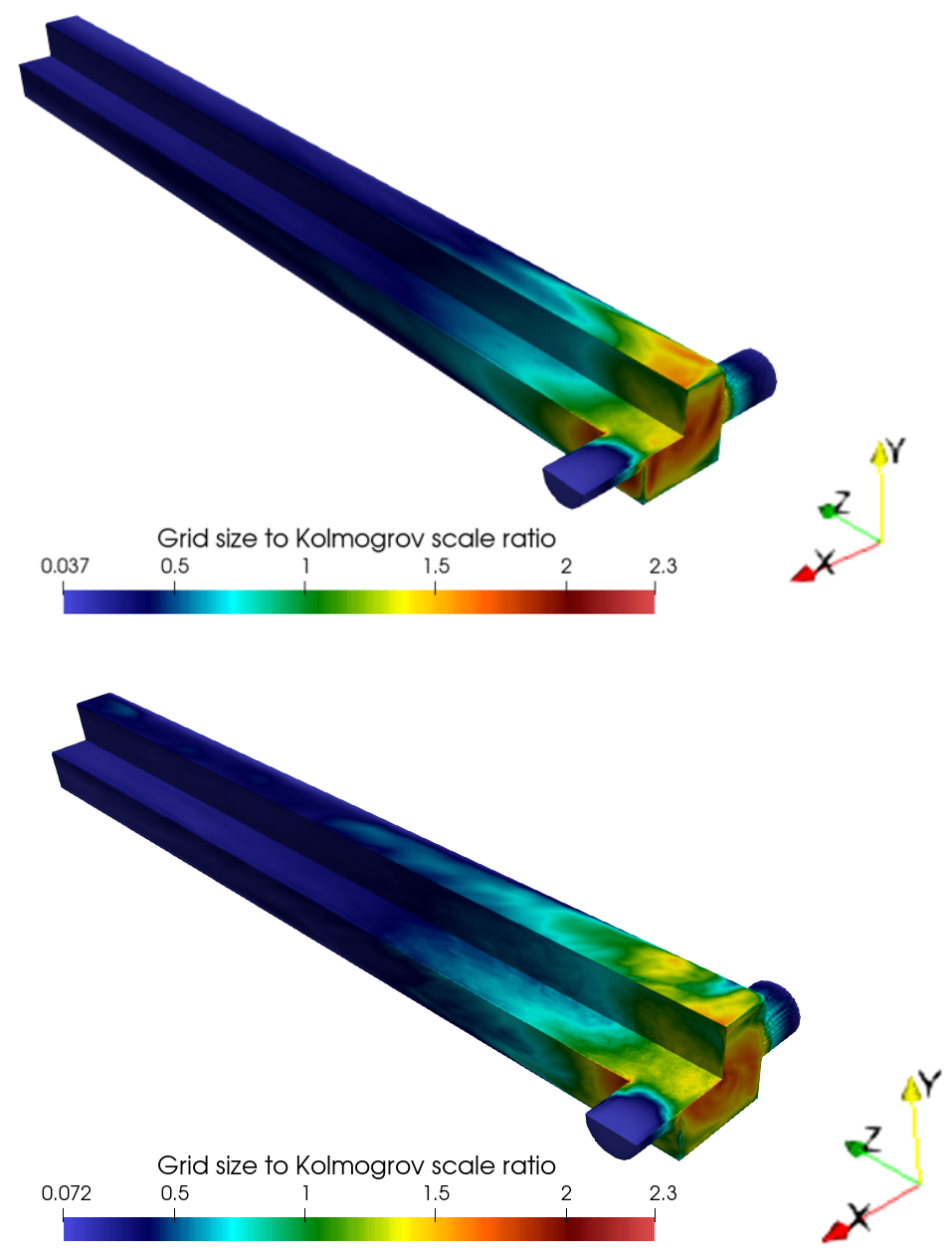

Figure 5: Grid spacing to Kolmogorov scale ratio in the flow-only simulation (top) and precipitation simulation (bottom).

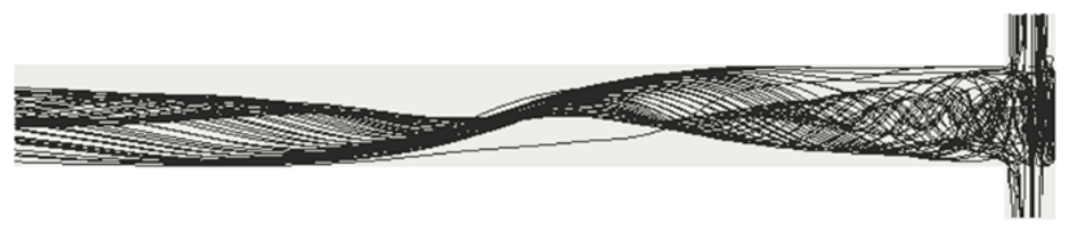

Figure 6: Streamlines of the mean flow. 
Figs. 8 and 9 show quantitative comparisons of the mean and root mean square (RMS) velocity predictions with the experimental measurements and DNS results reported in Schwertfirm et al. (2007). Good agreement is found between our simulation and the DNS results in that study in terms of both mean and RMS velocities. However, both simulations show the same discrepancy with respect to the PIV measurements. The major differences are in the mean $x$-component of the velocity over the mixing channel and the RMS $z$-component of the velocity over the feed. In Schwertfirm et al. (2007), these deviations were attributed mainly to uncertainties in the inflow conditions. In addition to that, the flow field may exhibit additional low frequency patterns (for example due to a precessing of the helical vortex) that may have not been reflected in the experimental measurements of mean and RMS velocity components. Further analysis of this motion, however, is beyond the scope of this paper.

Two key characteristics are worth pointing out: along the feed axis (direction indicated by the line in Fig. 7) in the impingement zone, we notice symmetric profiles in the RMS velocity components and the mean $z$ component of the velocity, while an asymmetric profile is observed in the mean $x$-component of the velocity. This implies the presence of the helical vortex as observed in Fig. 6 and 7. In addition, the mean and RMS velocity components decay along the downstream direction, suggesting that the turbulent intensity fades out shortly after the impingement of the two streams. Therefore, only the upstream of the mixing channel is dominated by convective mixing. Owing to this characteristic, the impingement area is expected to be the most dominant zone in the precipitation process. 


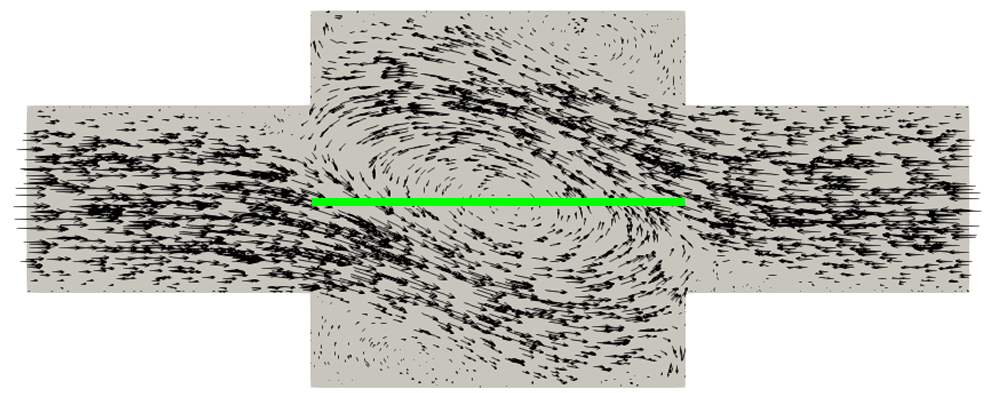

Figure 7: Mean velocity vector plot on the $\mathrm{X}-\mathrm{Y}$ plane at $\mathrm{Z}=0$.
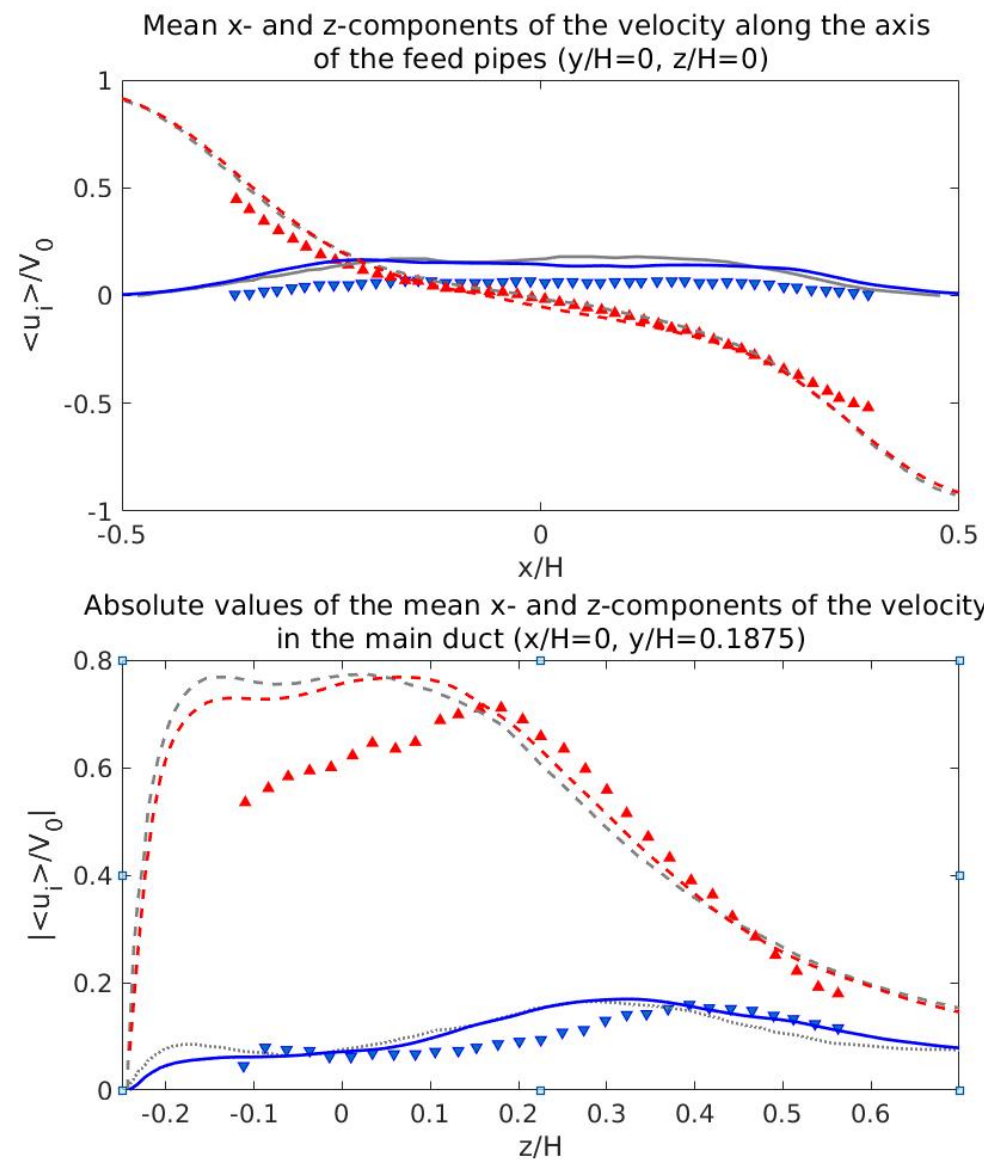

Figure 8: Normalised profiles of the mean $\mathrm{x}$ - (dashed line) and z- (solid line) components of the velocity along the axis of the feed pipes (top) and in the main duct (bottom) obtained by the DNS in the current work 28 coloured) and in Schwertfirm et al. (2007) (grey), compared with the experiments (triangle) in the latter. The results are normalised by the inlet peak velocity. 

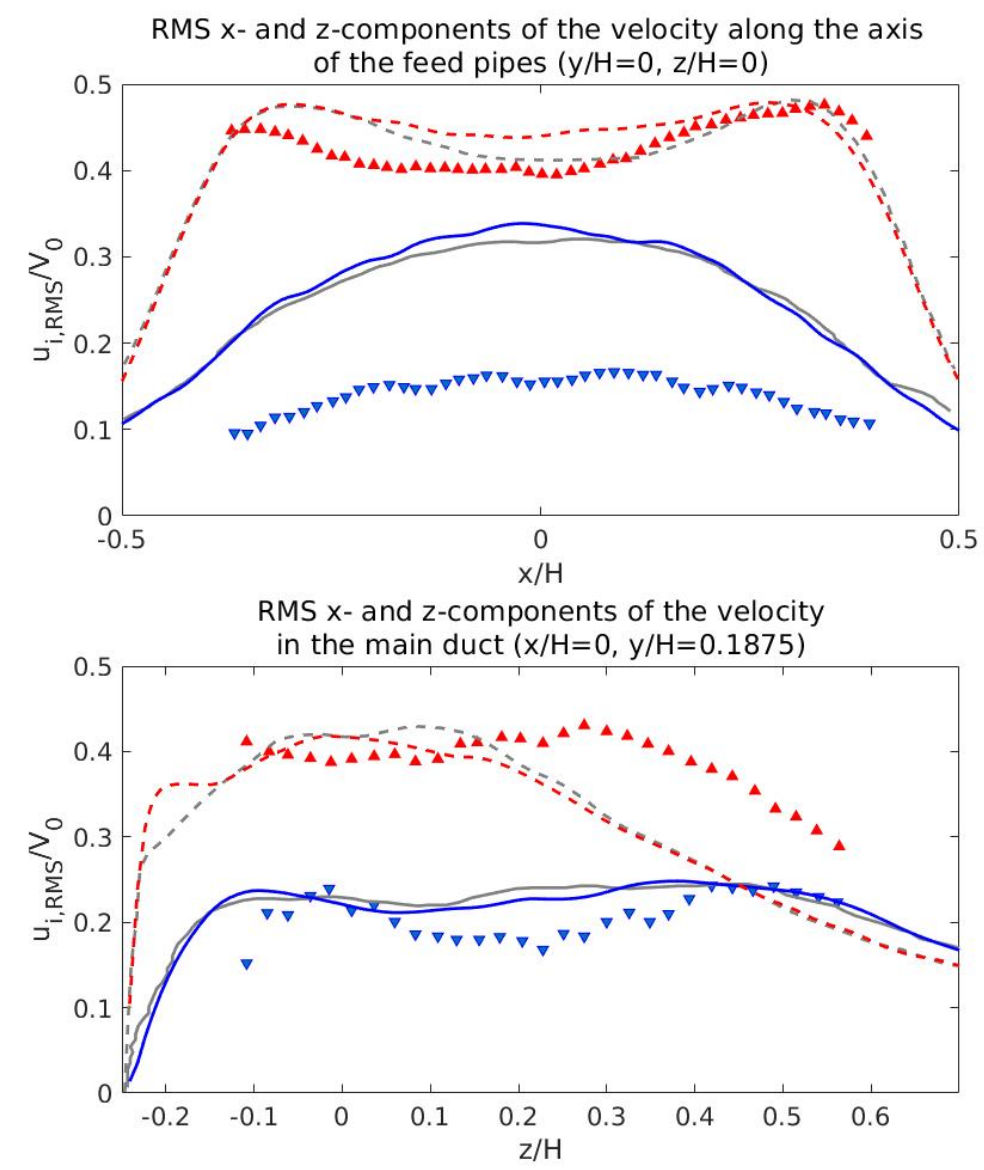

Figure 9: Normalised RMS profiles of the mean x- (dashed line) and z- (solid line) components of the velocity along the axis of the feed pipes (top) and in the main duct (bottom) obtained by the DNS from the current work (coloured) and from Schwertfirm et al. (2007) (grey), compared with the experiments (triangles) by the same authors. The results are normalised by the inlet peak velocity.

\subsection{Coupled DNS-PBE simulation}

In this section, we present the results of the precipitation simulation with the coupled DNS-PBE approach. The grid-size-to-Kolmogorov-scale ratio for this simulation is also shown in Fig. 5 and the comments in Sec. 4.2 about the 
grid resolution apply here too. Apart from the DNS resolution, two further issues had to be investigated to ensure that the numerics are accurate in this case. The first one is the convergence of the PBE grid, in order to ensure that the PSD is well resolved. The second one is the coupling of the source term between the species transport equations and the PBE. These issues will be discussed before presenting the DNS-PBE results.

\subsubsection{Convergence of PBE grid}

A convergence study of the composite PBE grid was carried out via a simulation in a perfectly mixed reactor. The inlet concentrations and residence time were identical with the T-mixer case. The result computed with a high-resolution geometric grid comprising $m=500$ intervals serves as a benchmark. The composite grid is also compared with a geometric grid with the same number of intervals, $m=45$. The comparison is shown in Fig. 10. Overall, the composite grid is capable of providing a distribution close to the benchmark result over a wide range of scales though the peak number density is slightly underpredicted. A certain amount of numerical diffusion is observed after $130 \mathrm{~nm}$, but the mean size is still predicted correctly, even at around $150 \mathrm{~nm}$. The accuracy in the fast growth zone is particularly good and almost matches with the benchmark case. In contrast, the $m=45$ geometric grid has poor resolution after $60 \mathrm{~nm}$ and the solution becomes diffusive before $100 \mathrm{~nm}$. The resulting 'spike-like' distribution fails to recreate the shape of the benchmark, and even the mean size begins to deviate when the PSD propagates to larger scales. In addition, the PSD given by the $m=45$ geometric grid exhibits a noticeable discrepancy in the nucleation zone (below $50 \mathrm{~nm}$ ). This occurs in spite of the fact that the geometric grid 
has a finer resolution than the composite grid in this size range, and is due to incorrect consumption of species induced by the coarse grid, which results in incorrect nucleation and growth rates. This can be evidenced from the supersaturation history (Fig. 11): the supersaturation level in the simulation with this grid is lower than in the other two, implying overestimated consumption of the reactant.
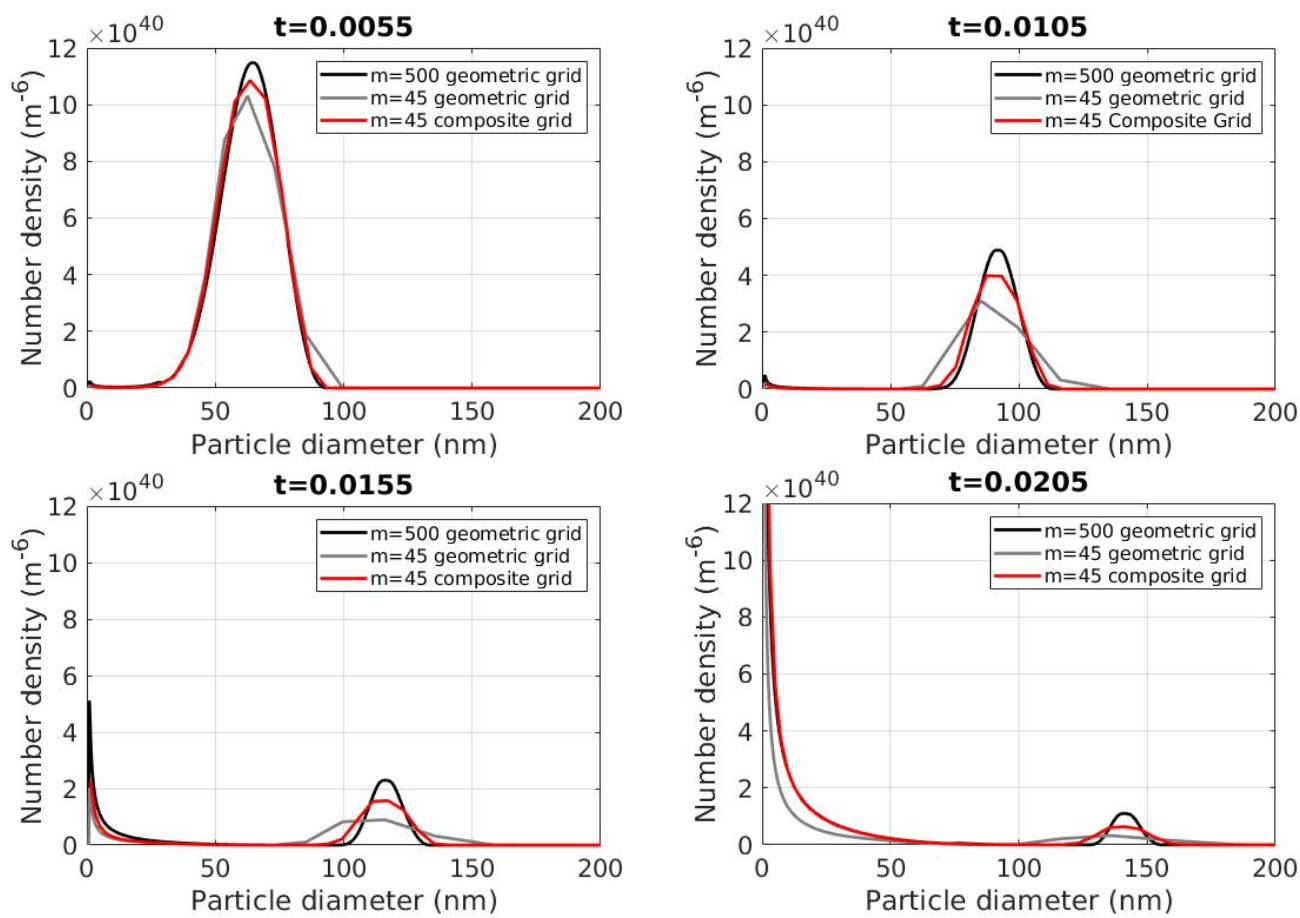

Figure 10: Convergence test on the composite grid. The PSD in a perfectly stirred reactor is computed and compared with a simulation featuring a geometric grid with the same number of nodes and one with a highly refined geometric grid. The results are presented at different time instances, at which the PSD has reached different size ranges. 


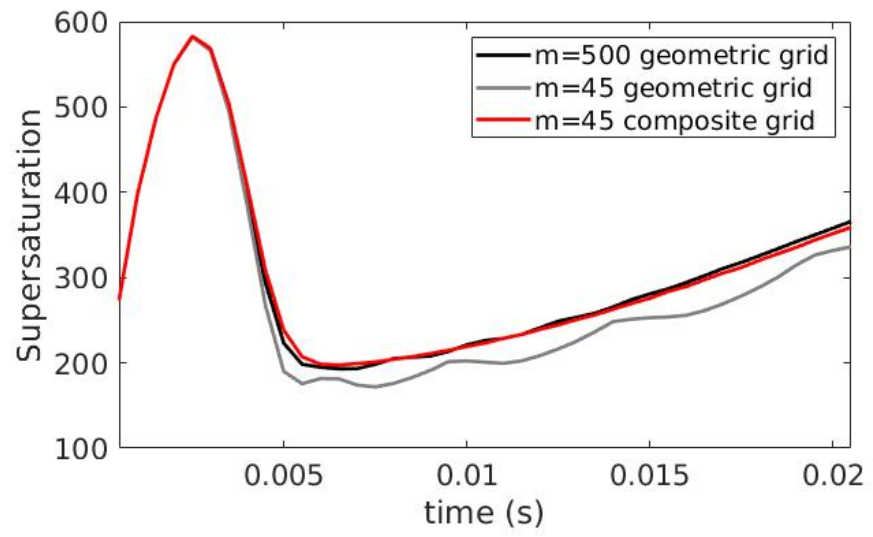

Figure 11: Evolution of supersaturation under different PBE grid in an ideal reactor.

\subsubsection{Validation of the source term coupling}

The coupling between the species transport equations and PBE must ensure conservation of mass during the exchange of mass between the particles formed and the species consumed. As explained in Sec. 2.3, in our simulation the volume of newly formed particles is obtained from the PSD via the rate of change of the first moment, which is then be converted to concentration of $\mathrm{BaSO}_{4}$. For the purpose of validation, the $\mathrm{BaSO}_{4}$ concentration was also computed from a transport equation using the reaction source term in Eq. 20. The resultant $\mathrm{BaSO}_{4}$ concentration is compared with the one obtained from the first moment. It was found that the average difference of $\mathrm{BaSO}_{4}$ concentration in the whole domain is less than $1 \%$ and the maximum local difference is below $6 \%$. Large discrepancies are found only at a few spots where steep gradients of the number densities are present, such as the boundaries between the impinging jets at the inlet. These differences are attributed to the discretisation in both physical and particle volume domains. Nevertheless, 
the difference found in the majority of the impingement zone and in the rest of the mixing channel remains small, implying a good coupling between the $\mathrm{PBE}$ and the generation of product concentration.

\subsubsection{Particle size distribution}

In the coupled DNS-PBE approach, it is possible to obtain the PSD at every point in the domain. In the experiment of Schwarzer et al. (2006), a measurement of the PSD was obtained at the outlet plane. A comparison of the time- and cross-sectional averaged PSD in the simulation with the one measured in the experiment, normalised by the zeroth moment of the distribution, is shown in Fig.12. The agreement is very good, apart from a slight overprediction of the peak. The particle size lies within the nanoparticle range, below $100 \mathrm{~nm}$. Most of the PSD lies within the uniform grid section, and therefore the composite grid provides good resolution.

The quality of the prediction allows us to evaluate the impact of various factors on modelling of turbulent precipitation. The flow field was fully resolved in this simulation. The discretisation approach allowed for solution of the PBE without the assumptions inherent in moment methods, and the composite grid allowed for good resolution of the PSD and minimal numerical diffusion. Finally, the direct coupling of an Eulerian PBE with fluid dynamics allowed for correct implementation of the source term, as opposed to the approach in Schwarzer et al. (2006), Gradl et al. (2006) and Gradl and Peukert (2009), where the PBE was applied as a post-processing step along Lagrangian fluid element trajectories in conjunction with the EDD mixing model. The kinetics are usually the main source of uncertainty in precipitation but, in this configuration, nucleation was limited to homogeneous 
nucleation, while there was no agglomeration due to the electrical charge of the ions. In different configurations, such as stirred tanks, heterogeneous nucleation may dominate, agglomeration and sometimes fragmentation may be present, and overall the kinetics are subject to more uncertainties. Finally, it must be noted that the DNS cannot resolve the Batchelor scales and the further mixing that occurs there. This effect was countered to some extent by the assumption of $\mathrm{Sc}=1$, which induces further mixing than the actual high Sc, although it is not possible with present (or foreseeable) resources to assess these effects with a DNS down to the Batchelor scales. However, the accuracy of this prediction indicates that the resolution of the flow field down to Kolmogorov scale, detailed solution of the PBE and direct coupling of PBE and DNS are the dominant factors in predicting turbulent precipitation, as long as the kinetics are reliable.

\section{Conclusions}

Turbulent precipitation is a very challenging problem to model, because it involves interactions between a turbulent flow field and non-linear processes such as nucleation and growth that feature timescales of an order of magnitude similar to turbulent mixing. A methodology for simulating turbulent precipitation and predicting the product PSD via coupling DNS and PBE has been presented. The DNS resolves the flow down to the Kolmogorov scale, while the PBE is solved via a discretisation method. A composite grid was proposed to provide sufficient resolution at the different size ranges covered by the distribution and to minimize numerical diffusion due to the particle growth term. In contrast to previous studies where the PBE was 


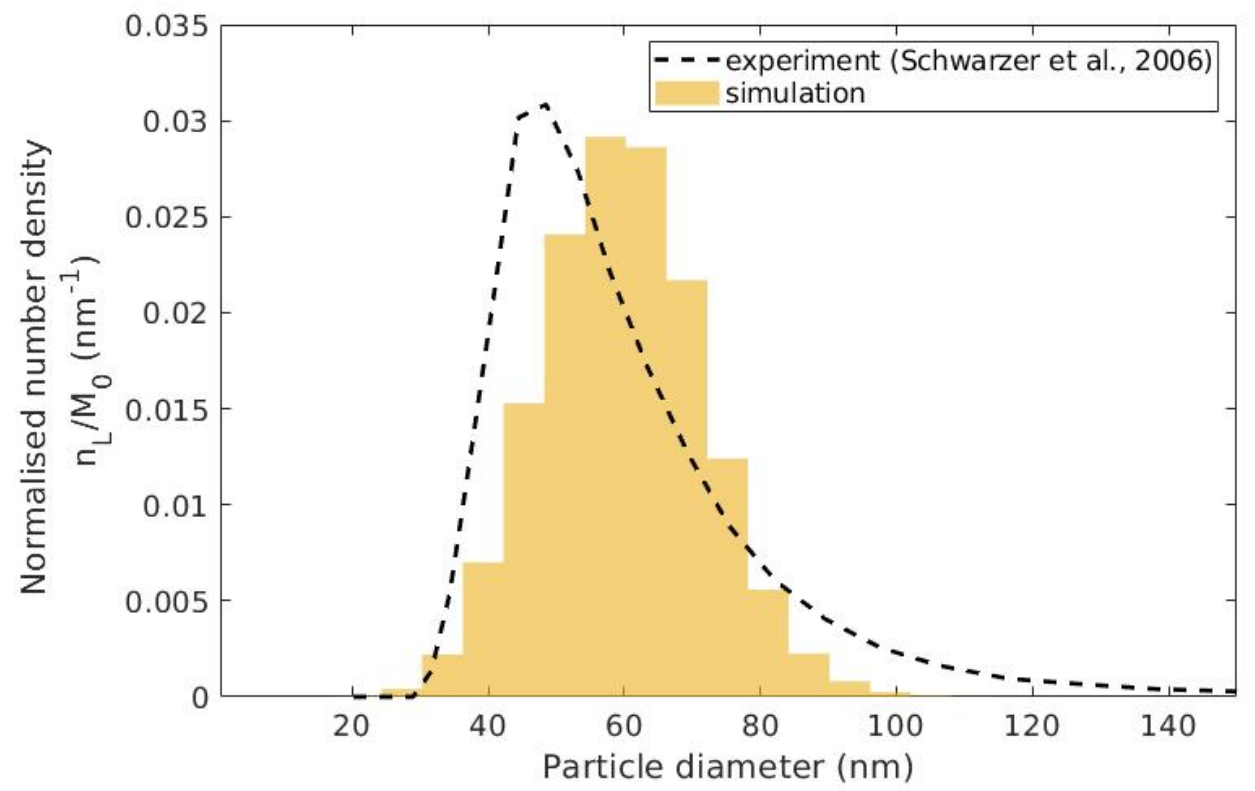

Figure 12: Time-and-plane-averaged PSD at outlet. Result is also compared with the measurement by Schwarzer et al. (2006)

applied as a post-processing step along a sample of Lagrangian trajectories, the coupling of the DNS and PBE is fully Eulerian in this study and the transport equations are directly coupled with the PBE via the source term. A CFL number for growth, analogous to the one for convection in physical space, was defined and it was ensured that the appropriate condition was satisfied.

The methodology was applied to the simulation of the experiments of Schwarzer et al. (2006) on $\mathrm{BaSO}_{4}$ nanoparticle precipitation. The numerical aspects of the method were first validated on their own: the flow field was validated with the PIV measurements on a scaled up geometry by Schwertfirm et al. (2007), the convergence of the composite grid for solution of 
the PBE was tested and the source term coupling was shown to satisfy the conservation of mass. In the DNS-PBE simulation, very good agreement with experimental results was obtained in terms of the outlet PSD. Classical theories were employed for the kinetics, and the level of agreement is partly owning to the homogeneous nucleation and lack of agglomeration in this case. While some assumptions are still involved, such as the Schmidt number effects, the results indicated that the resolution of the flow field and its direct coupling with the discretised PBE are of primary importance in simulating turbulent precipitation and prevent compensation of numerical and modelling errors. Future work will explore the potential of the methodology for drawing insight into the interaction of turbulence and particle formation in turbulent precipitation.

\section{Acknowledgements}

Hin Yan Tang acknowledges the financial support received from the Department of Mechanical Engineering, Imperial College London, in the form of a PhD studentship. Stelios Rigopoulos and George Papadakis acknowledge the financial support received from the Leverhulme Trust (grant RPG-2018101). The authors are grateful to the EPSRC (grants numbers: EP/R029369/1 and EP/R029326/1) and ARCHER for financial and computational support as a part of their funding to the UK Consortium on Turbulent Reacting Flows (www.ukctrf.com) and UK Turbulence Consortium (https://www.ukturbulence.co.uk/) respectively. We are also grateful to the UK Materials and Molecular Modelling Hub for computational resources on Thomas, through EPSRC grant $\mathrm{EP} / \mathrm{P} 020194 / 1$. 


\section{Nomenclature}

\section{Roman Letters}

$B \quad$ nucleation rate (as source of particle number density) $\quad m^{-6} s^{-1}$

$B_{N} \quad$ nucleation rate (as source of particle number) $\quad m^{-3} s^{-1}$

C concentration $\quad \mathrm{kmol} \mathrm{m}^{-3}$

$D$ diffusion coefficient $m^{2} s^{-1}$

$d_{i} \quad$ components of vector indicating distance between cell centroids $m$

$D_{A B}$ apparent diffusion coefficient $\quad m^{2} s^{-1}$

$G \quad$ volumetric growth rate $\quad m^{3} s^{-1}$

$G_{L} \quad$ linear growth rate $m s^{-1}$

$H$ mixer channel width / height m

$k \quad$ Boltzmann constant $J K^{-1}$

$k_{a} \quad$ surface shape factor $m$

$k_{d} \quad$ mass transfer coefficient $m \mathrm{~s}^{-1}$

$k_{v} \quad$ volume shape factor $m$

$K_{S P} \quad$ solubility product constant $\quad \mathrm{kmol}^{2} \mathrm{~m}^{-6}$

L particle size $m$ 
$L_{c} \quad$ nuclei size $m$

$m$ total number of intervals m

$M_{1} \quad$ first moment (total particle volume) per unit volume of solution $m$ $M W_{c}$ molecular weight of species in the crystal $\mathrm{kg} \mathrm{kmol}^{-1}$

$N$ number of particles $m$

$n$ face normal vector m

$n \quad$ number density (in particle volume) $\quad m^{-6}$

$N_{A} \quad$ Avogadro constant $\quad \mathrm{kmol}^{-1}$

$n_{L} \quad$ number density (in particle length) $\quad m^{-4}$

$R \quad$ reaction source $\quad k m o l m^{-3} s^{-1}$

$r \quad$ limiter variable m $m$

Re Reynolds number m

$S$ supersaturation ratio m

Sc Schmidt number $m$

Sh Sherwood number m

$T$ temperature $K$

$t$ time s s

$u \quad$ velocity $m s^{-1}$ 
$v \quad$ particle volume

$m^{3}$

$V_{0} \quad$ inlet peak velocity $m \mathrm{~s}^{-1}$

$V_{m} \quad$ molecular volume $m^{3}$

$v_{m, k} \quad$ particle volume at the midpoint of interval $\mathrm{k} \quad \mathrm{m}^{3}$

$x \quad$ spatial coordinate m

z charge number m

\section{Greek Letters}

$\beta_{m}$ blending coefficient m

$\Delta L_{k}$ distance between two neighbouring grid points in the PBE grid $m$

$\Delta x \quad$ Grid spacing $m$

$\gamma \quad$ blending factor $m$

$\gamma_{ \pm}$activity coefficient $m$

$\gamma_{C L}$ interfacial energy $J \mathrm{~m}^{-2}$

$\mu \quad$ dynamic viscosity $P a s$

$\nu \quad$ dissociation number $m$

$\nu_{\alpha} \quad$ stoichiometric coefficient of the $\alpha^{\text {th }}$ species $m$

$\phi \quad$ scalar quantity m

$\phi(r)$ limiter m $m$ 
$\rho \quad$ fluid density

$k g m^{-3}$

$\rho_{c} \quad$ crystal density

$k g m^{-3}$

$\tilde{\phi} \quad$ ratio of two consecutive differences (Eq. 9)

\section{Subscript}

$\alpha \quad$ species index

$m$

$D \quad$ downstream cell

$m$

$i \quad$ direction index

$m$

$k \quad$ interval index

$m$

$n \quad$ face normal

$m$

U upstream cell

$m$

\section{Superscript}

$+\quad$ right nodal point

$m$

- $\quad$ left nodal point

$m$

\section{References}

Abhyankar, S., Brown, J., Constantinescu, E.M., Ghosh, D., Smith, B.F., Zhang, H., 2018. Petsc/ts: A modern scalable ODE/DAE solver library. arXiv preprint arXiv:1806.01437 . 
Başbuğ, S., Papadakis, G., Vassilicos, J.C., 2018. Reduced power consumption in stirred vessels by means of fractal impellers. AIChE Journal 64, 1485-1499.

Balay, S., Abhyankar, S., Adams, M.F., Brown, J., Brune, P., Buschelman, K., Dalcin, L., Dener, A., Eijkhout, V., Gropp, W.D., Karpeyev, D., Kaushik, D., Knepley, M.G., May, D.A., McInnes, L.C., Mills, R.T., Munson, T., Rupp, K., Sanan, P., Smith, B.F., Zampini, S., Zhang, H., Zhang, H., 2019. PETSc Web page. https://www.mcs.anl.gov/petsc. URL: https://www.mcs.anl.gov/petsc.

Baldyga, J., Bourne, J., 1984a. A fluid mechanical approach to turbulent mixing and chemical reaction, Part II: Micromixing in the light of turbulence theory. Chemical Engineering Communications 28, 243-258.

Baldyga, J., Bourne, J., 1984b. A fluid mechanical approach to turbulent mixing and chemical reaction, Part III: Computational and experimental results for the new micromixing model. Chemical Engineering Communications 28, 259-281.

Bałdyga, J., Bourne, J.R., 1999. Turbulent Mixing and Chemical Reactions. Wiley.

Bałdyga, J., Orciuch, W., 1997. Closure problem for precipitation. Chemical Engineering Research and Design 75, 160-170.

Bałdyga, J., Orciuch, W., 2001. Barium sulphate precipitation in a pipe an experimental study and CFD modelling. Chemical Engineering Science $56,2435-2444$. 
Bouaniche, A., Vervisch, L., Domingo, P., 2019. A hybrid stochastic/fixedsectional method for solving the population balance equation. Chemical Engineering Science 209.

Derksen, J., 2012. Direct simulations of mixing of liquids with density and viscosity differences. Industrial and Engineering Chemistry Research 51, 6948-6957.

Di Veroli, G., Rigopoulos, S., 2009. A study of turbulence-chemistry interaction in reactive precipitation via a population balance-transported PDF method, in: Hanjalić, K., Nagano, Y., Jakirlić, S. (Eds.), Turbulence, Heat and Mass Transfer 6. Proceedings of the Sixth International Symposium on Turbulence, Heat and Mass Transfer, Rome, Italy, Begell House, Inc.

Di Veroli, G., Rigopoulos, S., 2010. Modeling of turbulent precipitation: A transported population balance-pdf method. AIChE Journal 56, 878-892.

Eble, A., 2000. Precpitation of nanoscale crystals with Perticular Reference to Interfacial Energy. Ph.D. thesis. TU Munchen.

Falgout, R.D., Yang, U.M., 2002. Hypre: A library of high performance preconditioners, in: Computational Science - ICCS 2002, Springer Berlin Heidelberg, Berlin, Heidelberg. pp. 632-641.

Fishpool, G.M., Leschziner, M.A., 2009. Stability bounds for explicit fractional-step schemes for the navier-stokes equations at high reynolds number. Computers and Fluids 38, 1289-1298.

Gao, F., O'Brien, E., 1992. A large-eddy simulation scheme for turbulent reacting flows. Physics of Fluids A 5, 1282-1284. 
Gavi, E., Marchisio, D., Barresi, A., 2007a. CFD modelling and scale-up of confined impinging jet reactors. Chemical Engineering Science 62, 22282241.

Gavi, E., Rivautella, L., Marchisio, D.L., Vanni, M., Barresi, A.A., Baldi, G., 2007b. CFD modelling of nano-particle precipitation in confined impinging jet reactors. Chemical Engineering Research and Design 85, 735-744.

Gradl, J., Peukert, W., 2009. Simultaneous 3D observation of different kinetic subprocesses for precipitation in a T-mixer. Chemical Engineering Science $64,709-720$.

Gradl, J., Schwarzer, H.., Schwertfirm, F., Manhart, M., Peukert, W., 2006. Precipitation of nanoparticles in a T-mixer: Coupling the particle population dynamics with hydrodynamics through direct numerical simulation. Chemical Engineering and Processing: Process Intensification 45, 908-916.

Gunawan, R., Fusman, I., Braatz, R.D., 2004. High resolution algorithms for multidimensional population balance equations. AIChE Journal 50, 2738-2749.

Hulburt, H.M., Katz, S., 1964. Some problems in particle technology. A statistical mechanical formulation. Chemical Engineering Science 19, 555574 .

Jasak, H., Weller, H.G., Gosman, A.D., 1999. High resolution NVD differencing scheme for arbitrarily unstructured meshes. International Journal for Numerical Methods in Fluids 31, 431-449. 
Koren, B., 1993. A robust upwind discretization method for advection, diffusion and source terms. Vieweg, Germany.

Kucher, M., Babic, D., Kind, M., 2006. Precipitation of barium sulfate: Experimental investigation about the influence of supersaturation and free lattice ion ratio on particle formation. Chemical Engineering and Processing: Process Intensification 45, 900-907.

Liu, A., Rigopoulos, S., 2019. A conservative method for numerical solution of the population balance equation, and application to soot formation. Combustion and Flame 205, 506-521.

Makowski, T., Bałdyga, J., 2011. Large eddy simulation of mixing effects on the course of parallel chemical reactions and comparison with $\mathrm{k}-\epsilon$ modeling. Chemical Engineering and Processing: Process Intensification 50, 10351040.

Marchisio, D.L., Fox, R.O., 2005. Solution of population balance equations using the direct quadrature method of moments. Journal of Aerosol Science $36,43-73$.

Marchisio, D.L., Rivautella, L., Barresi, A.A., 2006. Design and scale-up of chemical reactors for nanoparticle precipitation. AIChE Journal 52, 1877-1887.

McGraw, R., 1997. Description of aerosol dynamics by the quadrature method of moments. Aerosol Science and Technology 27, 255-265.

Mersmann, A. (Ed.), 2001. Crystallization Technology Handbook, Second Edition. Marcel Dekker. 
Metzger, L., Kind, M., 2017. The influence of mixing on fast precipitation processes - a coupled 3D CFD-PBE approach using the direct quadrature method of moments (DQMOM). Chemical Engineering Science 169, 284298.

Moin, P., Mahesh, K., 1998. Direct numerical simulation: A tool in turbulence research. Annual Review of Fluid Mechanics 30, 539-578.

Myerson, A.S., Erdemir, D., Lee, A.Y. (Eds.), 2019. Handbook of Industrial Crystallization, Third Edition. Cambridge University Press.

Paul, I., Papadakis, G., Vassilicos, J.C., 2018. Direct numerical simulation of heat transfer from a cylinder immersed in the production and decay regions of grid-element turbulence. Journal of Fluid Mechanics 847, 452-488.

Qamar, S., Elsner, M.P., Angelov, I.A., Warnecke, G., Seidel-Morgenstern, A., 2006. A comparative study of high resolution schemes for solving population balances in crystallization. Computers and Chemical Engineering 30, 1119-1131.

Qamar, S., Warnecke, G., Elsner, M.P., 2009. On the solution of population balances for nucleation, growth, aggregation and breakage processes. Chemical Engineering Science 64, 2088-2095.

Rhie, C.M., Chow, W.L., 1983. Numerical study of the turbulent flow past an airfoil with trailing edge separation. AIAA Journal 21, 1525-1532.

Rigopoulos, S., 2007. PDF method for population balance in turbulent reactive flow. Chemical Engineering Science 62, 6865-6878. 
Rigopoulos, S., 2019. Modelling of soot aerosol dynamics in turbulent flow. Flow, Turbulence and Combustion .

Rigopoulos, S., Jones, A., 2001. Dynamic modelling of a bubble column for particle formation via a gas-liquid reaction. Chemical Engineering Science $56,6177-6184$.

Rigopoulos, S., Jones, A., 2003a. A hybrid CFD-reaction engineering framework for multiphase reactor modelling: Basic concept and application to bubble column reactors. Chemical Engineering Science 58, 3077-3089.

Rigopoulos, S., Jones, A., 2003b. Modeling of semibatch agglomerative gasliquid precipitation of $\mathrm{CaCO} 3$ in a bubble column reactor. Industrial and Engineering Chemistry Research 42, 6567-6575.

Schubert, H., 1998. Keimbildung bei der Kristallisation schwerlöslicher Feststoffe. Ph.D. thesis. Technische Univetsität München.

Schwarzer, H.., Schwertfirm, F., Manhart, M., Schmid, H.., Peukert, W., 2006. Predictive simulation of nanoparticle precipitation based on the population balance equation. Chemical Engineering Science 61, 167-181.

Schwarzer, H.C., Peukert, W., 2002. Experimental investigation into the influence of mixing on nanoparticle precipitation. Chemical Engineering and Technology 25, 657-661.

Schwarzer, H.C., Peukert, W., 2004a. Combined experimental/numerical study on the precipitation of nanoparticles. AIChE Journal 50, 3234-3247. 
Schwarzer, H.C., Peukert, W., 2004b. Tailoring particle size through nanoparticle precipitation. Chemical Engineering Communications 191, 580-606.

Schwarzer, H.C., Peukert, W., 2005. Prediction of aggregation kinetics based on surface properties of nanoparticles. Chemical Engineering Science 60, $11-25$.

Schwertfirm, F., Gradl, J., Schwarzer, H.C., Peukert, W., Manhart, M., 2007. The low reynolds number turbulent flow and mixing in a confined impinging jet reactor. International Journal of Heat and Fluid Flow 28, 14291442.

Sewerin, F., Rigopoulos, S., 2017a. An explicit adaptive grid approach for the numerical solution of the population balance equation. Chemical Engineering Science 168, 250-270.

Sewerin, F., Rigopoulos, S., 2017b. An LES-PBE-PDF approach for modeling particle formation in turbulent reacting flows. Physics of Fluids 29.

Sewerin, F., Rigopoulos, S., 2018. An LES-PBE-PDF approach for predicting the soot particle size distribution in turbulent flames. Combustion and Flame 189, 62-76.

Sewerin, F., Rigopoulos, S., 2019. Algorithmic aspects of the LES-PBE-PDF method for modeling soot particle size distributions in turbulent flames. Combustion Science and Technology 191, 766-796.

Söhnel, O., Garside, J., 1992. Handbook of Industrial Crystallization. Butterworth-Heinemann Ltd. 
Telib, H., Manhart, M., Iollo, A., 2004. Analysis and low-order modeling of the inhomogeneous transitional flow inside a T-mixer. Physics of Fluids 16, 2717-2731.

Thomareis, N., Papadakis, G., 2017. Effect of trailing edge shape on the separated flow characteristics around an airfoil at low Reynolds number: A numerical study. Physics of Fluids 29, 014101.

Thomareis, N., Papadakis, G., 2018. Resolvent analysis of separated and attached flows around an airfoil at transitional Reynolds number. Phys. Rev. Fluids 3, 073901.

van Vliet, E., Derksen, J., van den Akker, H., Fox, R., 2007. Numerical study on the turbulent reacting flow in the vicinity of the injector of an ldpe tubular reactor. Chemical Engineering Science 62, 2435 - 2444.

Villermaux, J., 1989. A simple model for partial segregation in a semibatch reactor, in: AIChE Meeting San Francisco, Paper 114a.

van Vliet, E., Derksen, J.J., van den Akker, H.E.A., 2005. Turbulent mixing in a tubular reactor: Assessment of an fdf/les approach. AIChE Journal $51,725-739$.

Wu, B., Fang, Y., Zhao, C., Wang, Y., Luo, P., 2017. Experimental study and numerical simulation of barium sulfate precipitation process in a continuous multi-orifice-impinging transverse jet reactor. Powder Technology $321,180-189$.

Xiao, D., Papadakis, G., 2017. Nonlinear optimal control of bypass transition in a boundary layer flow. Phys. Fluids 29, 054103. 
Xiao, D., Papadakis, G., 2019. Nonlinear optimal control of transition due to a pair of vortical perturbations using a receding horizon approach. Journal of Fluid Mechanics 861, 524-555.

Zauner, R., Jones, A., 2000. Determination of nucleation, growth, agglomeration and disruption kinetics from experimental precipitation data: The calcium oxalate system. Chemical Engineering Science 55, 4219-4232. 